\title{
Stimulus processing in autonomously active cognitive systems
}

\author{
Claudius Gros \\ Institute of Theoretical Physics, J.W. Goethe University \\ 60054 Frankfurt/Main, Germany
}

\begin{abstract}
The brain is autonomously active and possesses an ongoing internal dynamics which continues even in the temporary absence of external sensory stimuli. New experimental evidences, and theoretical considerations, indicate that this eigendynamics plays a central role in regulating the overall cognitive processing, a key property which is expected to be true also for prospective artificial cognitive systems, capable of synthetic generalized intelligence.

It is therefore of paramount importance to study possible frameworks for autonomously active cognitive systems. We report here the status of research for an approaches based on transient state dynamics and give an overall view of the embedding of this new paradigm within mainstream approaches in artificial intelligence and systems neuroscience.
\end{abstract}

\section{What is an autonomously active cognitive systems?}

Mainstream research both in artificial intelligence as well as in robotics involves task solving (Konar 2005), like hand-writing recognition, face recognition in pictures or autonomous navigation by robotic cars a la DARPA Grand Challenge. Tremendous progress has been reported for many computational tasks and its a long time that top human chess players had been able to beat the best chess programs.

In this context the notion of 'autonomy' is usually used in two different connotations. Autonomous robots tackle the tasks given without external help, making use of their own sensors and their previous programming. It is often assumed implicitly, that a steady increase in the complexity and in the versatility of autonomous robots could, in the long-time perspective, eventually lead to human-level universal artificial intelligence (AI).

There is an alternative view to this route to a general artificial intelligence which is motivated both by theory considerations and by recent advances in neurobiology (Gros 2009a; 2009b). In this view the term 'autonomy' has a different denotation. It is well known

Copyright (C) 2008, The Second Conference on Artificial General Intelligence (AGI-09.org). All rights reserved. that the brain is autonomously active in the sense that non-trivial cognitive neural activity persists even in the prolonged absence of sensory input. The brain has the task to process the sensory data input stream it receives in order to keep the body alive and functional, in a dynamic environment. The question is then, whether the autonomous eigendynamics is just a side effect, an epiphenomena, of the interwoven activity of the constituing cognitive modules. Alternatively it is possible that the eigendynamics has a central regulating role.

In this second view cognitive capabilities evolve through the mutual interaction between the autonomous neural eigendynamics and the input the brain receives from the sensory organs. Higher algorithmic task solving capabilities are not genetically prewired but evolve throughout the development of the growing animal or the synthetic intelligence.

The self-sustained neural activity of the brain is in this view a conditio sine qua non. Higher cognitive capabilities would neither evolve in the absence of this autonomous dynamics nor would they be present at birth. They are not encoded explicitly in the genes. The internal autonomous neural dynamics has, as a corollary of this view, at birth no semantic content, since semantic significance of neural activity patterns can arise only in conjunction with environmental information.

\section{Modelling principles}

At the basis of this concept of autonomously active cognitive systems lies the formulation of the internal selfsustained dynamical activity, for which various governing principles have been proposed (Gros 2009a). Our own approach is based on the notion of transient neural states (Gros 2007), illustrated in Fig. 1, in part motivated by experimental observations (Abeles et al. 1995; Kenet et al. 2003). The time scale of the transiently stable neural activity relates to the typical cognitive time scale of about $80-100 \mathrm{~ms}$. Transient state dynamics can be cast, within dynamical system theory (Gros $2008)$, into the framework of an attractor relic network (Gros 2007; 2009a).

The internal dynamics of an autonomously active cognitive system is not driven by the sensory input. Identical stimuli will generally lead to different re- 


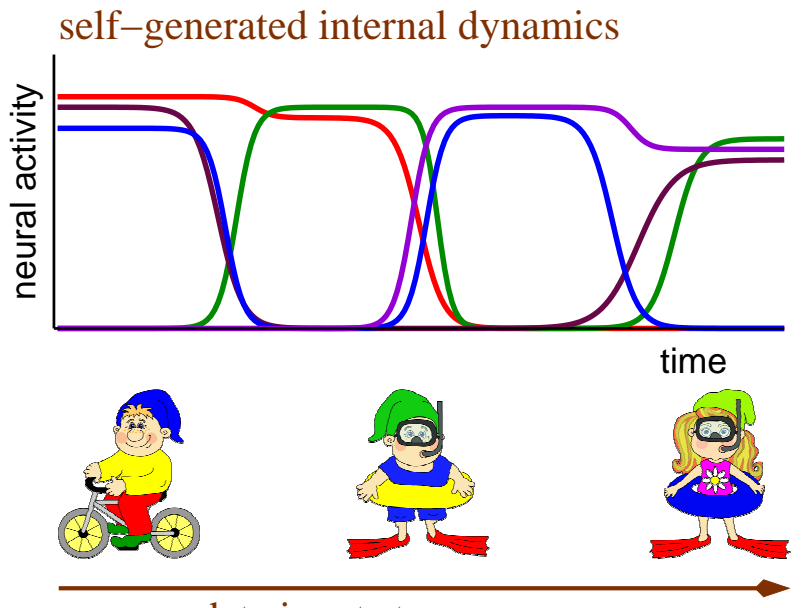

sensory data input stream

Figure 1: Cartoon of an autonomously active cognitive system. The self-generated eigendynamics in terms of a time series of transient states (top). This dynamical activity is a priori not related to the sensory data input stream (bottom), carrying environmental information. Emergent cognitive capabilities need to result from the emergence of correlation between the internal activity and the sensory data stream through unsupervised online learning.

sponses, or to no response at all, depending on the currently ongoing internal activity (Arieli et al. 1996). This state of affairs may be interpreted from two different perspectives. On one side one may view the brain as a finite-state machine for which the next state depends both on the current state and on the input. From a neurobiological perspective one may regard on the other side the brain as an autonomously active system for which the internal dynamics is modulated, but not forcefully driven, by external stimuli.

The second viewpoint allows to formulate a simple and yet very powerful principle governing the influence of the sensory data stream onto the internal activity. The internal transient-state dynamics is realized by competitive neural processes. This competition can be influenced and modulated by the sensory input when we assume that the sensory signals contribute to the internal neural competition on an equal basis. This principle has been implemented successfully (Gros \& Kaczor 2008) and tested for the bars problem. The bars problem is a standardized setup for a non-linear independent component task.

The exciting result is now the following (Gros \& Kaczor 2008; Gros 2009a): We have a generalized neural network which is continuously and autonomously active on its own. There is no external teacher and no explicit coding of any algorithm, all learning rules are online and Hebbian-style. In this setup the competition of the internal, autonomously generated transient state dynamics, with the data input stream leads to an emergent cognitive capability, an independent component analysis. As a result of this process the internal transient states, the attractor relics, are mapped via their respective receptive fields to objects present in the environment, the independent components. The internal transient state dynamics acquires such a semantic content and turns into an associative thought process.

\section{Conclusions}

This is a non-technical position paper and we refer to the literature both for details on our own work (Gros 2007 ; 2009b), as well as for a review on the experimental situation and on alternative approaches (Gros 2009a). These results demonstrate the feasibility of the basic concept, the emergence of cognitive capabilities through the interaction of the internal eigendynamics and the external influences arriving via the sensory organs. It is our believe that the field of autonomously active cognitive system constitutes a field of emergent importance, both for systems neuroscience as well as for the eventual development of general artificial intelligences.

\section{References}

Abeles, M.; Bergman, H.; Gat, I.; Meilijson, I.; Seidemann, E.; Tishby, N.; and Vaadia, E. 1995. Cortical Activity Flips Among Quasi-Stationary States. Proceedings of the National Academy of Sciences 92:86168620 .

Arieli, A.; Sterkin, A.; Grinvald, A.; and Aertsen, A. 1996. Dynamics of Ongoing Activity: Explanation of the Large Variability in Evoked Cortical Responses. Science 273:1868.

Gros, C., and Kaczor, G. 2008. Learning in cognitive systems with autonomous dynamics. In Proceedings of the International Conference on Cognitive Systems, Karlsruhe. Springer.

Gros, C. 2007. Neural networks with transient state dynamics. New Journal of Physics 9:109.

Gros, C. 2008. Complex and Adaptive Dynamical Systems: A Primer. Springer.

Gros, C. 2009a. Cognitive computation with autonomously active neural networks: an emerging field. Cognitive Computation. (in press).

Gros, C. 2009b. Emotions, diffusive emotional control and the motivational problem for autonomous cognitive systems. In Handbook of Research on Synthetic Emotions and Sociable Robotics: New Applications in Affective Computing and Artificial Intelligence. J. Vallverdu, D. Casacuberta (Eds.), IGI-Global. (in press). Kenet, T.; Bibitchkov, D.; Tsodyks, M.; Grinvald, A.; and Arieli, A. 2003. Spontaneously emerging cortical representations of visual attributes. Nature 425:954956.

Konar, A. 2005. Computational Intelligence: Principles, Techniques And Applications. Springer. 